\title{
UNCERTAINTY PROPAGATION FOR LWR BURNUP BENCHMARK USING SAMPLING BASED CODE SCALE/SAMPLER
}

\author{
DANiEl CAmpolina $^{a, b, *}, \mathrm{JAN}_{\mathrm{FRyBORT}}{ }^{a}$ \\ ${ }^{a}$ Department of Nuclear Reactors, Czech Technical University in Prague, Prague, Czech Republic \\ ${ }^{b}$ Nuclear Technology Development Center, Belo Horizonte, Brazil \\ * corresponding author: campolina@cdtn.br
}

\section{ABSTRACT.}

Sampling based method is adopted in many fields of engineering and it is currently used to propagate uncertainties from physical parameters and from nuclear data, to integral indicators of nuclear systems. The total uncertainty associated with a model simulation is of major importance for safety analysis and to guide vendors about acceptable tolerance limits for nuclear installations parts. This work presents some calculations to propagate uncertainties for a nuclear reactor fuel element modeled in SCALE/TRITON, using the sampling tool SCALE/SAMPLER. Results showed that the influence of input uncertainties on $k_{\mathrm{inf}}$ is more pronounced in the fresh core other than the depleted core and the contribution from studied manufacturing uncertainties is smaller than the contribution of nuclear data uncertainties.

KEYWORDS: random sampling, uncertainty, nuclear fuel burnup, OECD UAM LWR.

\section{INTRODUCTION}

In a global picture, uncertainty quantification (UQ) is the process of characterizing input uncertainties, forward propagating these uncertainties through a computational model, and performing statistical assessments on the resulting responses. In this forward propagation, illustrated in Fig, 1. probabilistic or interval information on parametric inputs are mapped through the computational model to assess statistics or intervals on outputs. The computational model for the present application is the TRITON control module of SCALE, composed of cross-section processing codes, a neutron transport solver, and point depletion code [1].

For UQ, components of the input parameter are considered to be uncertain as specified by probability distributions (e.g., normal, beta). By stipulating specific distributional structure to the inputs, distributional structure for the outputs (i.e, response statistics) can be inferred [2]. Uncertainties and correlations among experimentally measured cross-sections constitute the so called nuclear data covariance libraries. The sampling based approach can be used to sample physical parameters like dimensions and densities and also joint probability density functions given in the nuclear data covariance libraries. The last produces a random sample for the nuclear cross-sections that are used in a transport calculation.

One of the most important results of a nuclear reactor simulation is neutron multiplication factor (k) that measures the balance between production of neutrons and their absorption in the core and leakage out of the core. The multiplication factor is a key

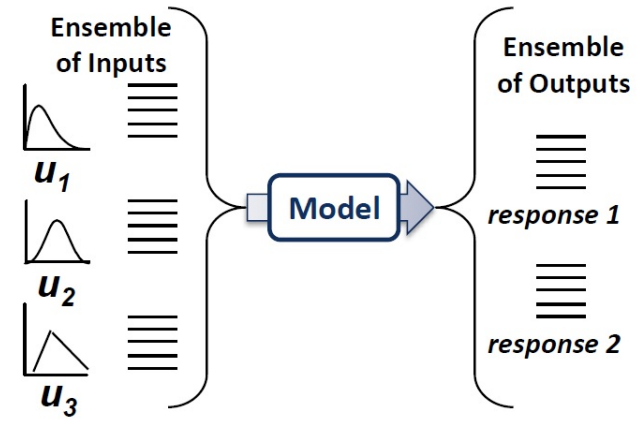

FiguRE 1. Realizations from specified input distributions being run in a model simulation.

indicator for the changes in the neutron flux that also causes changes in the power level of the reactor. If the multiplication factor is lower than 1 , the system is subcritical and its power is decreasing. Loss of neutrons is greater than their production and it is impossible to maintain the fission chain reaction.

A UQ analysis can provide the level of confidence in the multiplication factor predicted by simulations and identify through a sensitivity analysis the key parameters whose uncertainties contribute most to the multiplication factor uncertainty. There are always safety margins to multiplication factor in criticality calculations. It reflects the uncertainty in all calculations. When subcriticality of some system must be certified the calculations are usually aimed at maximum multiplication factor by $5 \%$ below the criticality. The UQ process can verify that such a margin is sufficient for any design systems.

Since sampling based method only requires forward 
calculations, it presents some advantages if compared to adjoint-based perturbation theory approach used by e.g. TSUNAMI modules of SCALE. In problems with significant second order effects, the first order perturbation theory is not valid while the sampling method is fully applicable. The typical example of the second order effect is when the perturbation in the transport operator causes significant perturbation in the flux solution of the Boltzmann transport equation (see chapter 6 of [1]).

SCALE/SAMPLER is one of the sampling tools available at Department of Nuclear Reactors of CVUT for uncertainty qualification. It is at first used in this study to assess the impact of covariance data on nuclear transport and depletion of a LWR Benchmark 2D model. Then effect of geometric uncertainties, also known as manufacturing uncertainties, are quantified for the same model.

\section{UNCERTAINTY ANALYSIS FROM NUCLEAR DATA}

In this section, cross-section perturbation using SCALE/SAMPLER is performed for a benchmark proposed by the OECD expert group Uncertainty Analysis in Modeling (UAM) [3]. Resulted values of propagated uncertainties to $k_{\text {inf }}$ are compared against participant-averaged results for the UAM benchmark.

\subsection{Methodology}

The UAM benchmark has several exercises. This analysis is using fuel pin model of a typical PWR fuel rod in $15 \times 15$ assembly design from exercise I-b. This was proposed in scope of the UAM benchmark in order to address uncertainties in nuclear fuel depletion calculation due to the basic nuclear data as well as the impact of processing the nuclear and covariance data. Summary and discussion of neutronic cases performed by the participants is available in [4]. Participantaveraged results for exercise I-b are reproduced here for comparison purposes despite different input covariance cross-sections were used by the participants. The UAM expert group selected 44-group SCALE 6.0 covariance library as a reference library for the benchmark exercise since it was the most complete and up-to-date compilation at the time of that review.

Calculations in SCALE are conducted according to control modules and sequences. This study adopted T-DEPL sequence of TRITON that couples 2D multigroup deterministic transport in NEWT with ORIGEN module depletion.

The lattice physics calculations are based on the 56-group energy structure of the v7-56 SCALE library. This library is recommended for light-water reactor calculations. Trace amount of 231 nuclides was added in the inventory of burnable materials (addnux $=3$ card of TRITON) in order to reach more realistic fuel depletion from the very beginning. Infinite lattice of the elementary cell was simulated by reflective boundary condition adopted for all sides of the model.

\begin{tabular}{lc}
\hline Parameter & Value \\
\hline HFP conditions & \\
Fuel temperature $(\mathrm{K})$ & 900 \\
Cladding temperature $(\mathrm{K})$ & 600 \\
Moderator (coolant) temperature $(\mathrm{K})$ & 562 \\
Moderator (coolant) density $\left(\mathrm{g} / \mathrm{cm}^{3}\right)$ & 0.7484 \\
Configuration & \\
Unit cell pitch $(\mathrm{mm})$ & 14.427 \\
Fuel pellet diameter $(\mathrm{mm})$ & 9.391 \\
Fuel pellet material & $\mathrm{UO}$ \\
Fuel density (g/cm $\left.{ }^{3}\right)$ & 10.283 \\
Fuel enrichment $(\mathrm{w} / \mathrm{o})$ & 4.85 \\
Cladding outside diameter $(\mathrm{mm})$ & 10.928 \\
Cladding thickness $(\mathrm{mm})$ & 0.673 \\
Cladding material & Zircaloy-4 \\
Cladding density $\left(\mathrm{g} / \mathrm{cm}^{3}\right)$ & 6.55 \\
Gap material & $\mathrm{He}$ \\
Moderator material & $\mathrm{H}_{2} \mathrm{O}$ \\
Operating history & \\
Operating cycle & 1 \\
Burn time (days) & 1825 \\
Final Burn-up $(\mathrm{GWd} / \mathrm{MTU})$ & 61.28 \\
Specific power $(\mathrm{kW} / \mathrm{kgU})$ & 33.58 \\
\hline
\end{tabular}

TABle 1. Configuration and conditions of burnup pin-cell test problem.

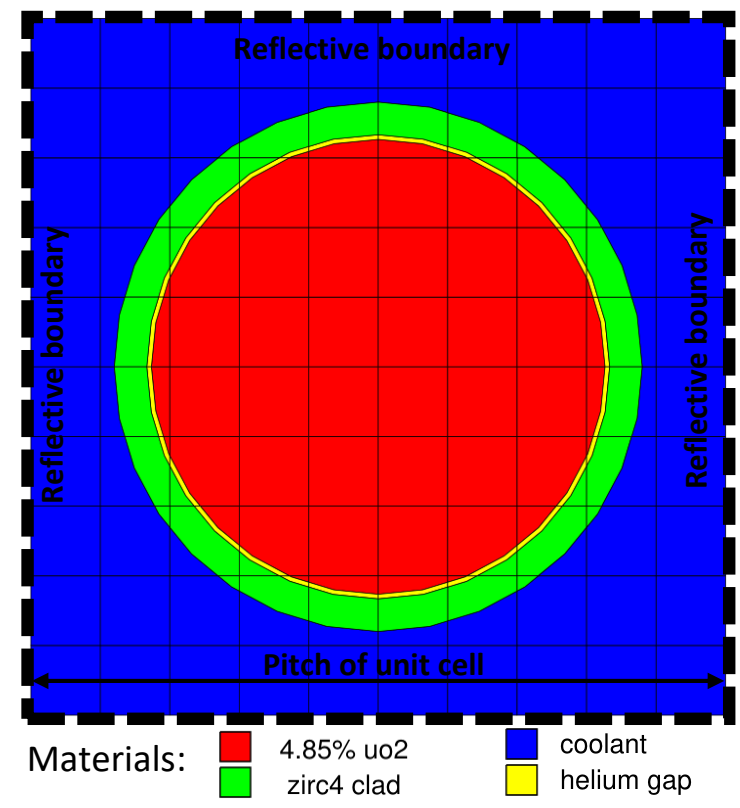

Figure 2. TRITON model model for Exercise I-1b Pin Cell.

The SCALE/SAMPLER module was used to perform uncertainty propagation/analysis of neutron multiplication factor for the pin-cell shown in Fig. 2, Data library for cross-section perturbations is described in the following section.

\section{Cross section COVARIANCE LIBRARY}

The new 56-group (56groupcov) SCALE 6.2 covariance library [1], recommended by SCALE 6.2.1 for 


\begin{tabular}{c|c|ccc}
\hline$k_{\text {inf }}$ & 0 GWd/MTU & \multicolumn{3}{|c}{60 GWd/MTU } \\
& SAMPLER & SAMPLER & Ref.* & $\Delta$ \\
\hline Mean val & 1.4023 & 0.9063 & 0.9030 & $0.4 \%$ \\
RSD (\%) & 0.55 & 0.58 & 0.89 & - \\
SD (pcm) & 770 & 529 & 805 & $52 \%$ \\
\hline
\end{tabular}

TABLE 2. Initial condition and final burnup uncertainties in $k_{\text {inf }}$ for cases UAM* $^{*}$ (using 44groupcov) and SAMPLER (using 56groupcov).

all applications, was used by SAMPLER uncertainty module. The data correspond to relative uncertainties assembled from a variety of sources, including highfidelity covariance evaluations from ENDF/B-VII.1 [5] as well as approximate uncertainties obtained from the collaborative projects involving BNL, LANL, and ORNL. The assumptions in generating the data, the library contents, and processing procedure for the SCALE 56-group covariance libraries can be found in 1]. Comparison between the two libraries adopted by SAMPLER and adopted by UAM participants results are provided in next section in terms of relative standard deviation for some reactions, in order to clarify the difference and the probable impact on results.

The number of samples/realizations in SAMPLER code was set to 1000, that is the maximum allowed for cross section sampling. Recent works show that 1000 calculations are enough to permit optimal convergence of the statistical moments of significance for any given response function 6 . 8 .

It is important to emphasize that only cross-section (XS) perturbation is performed and it is the most important contributor to the overall uncertainty. Neither fission yield perturbation nor decay data perturbations are performed. Some studies considering the same benchmark concluded that contribution of decay data uncertainty for burnup calculations is negligible [9. On the other hand, other studies stated that fission yield and decay data uncertainties may be relevant for uncertainties of nuclide densities in depletion calculations [10].

\subsection{Results}

Propagation of uncertainties to initial and final burnup results performed using SAMPLER are presented in Tab. 2 together with the mean value of $k_{\text {inf }}$ calculated. Participant-averaged result from exercise I-b benchmark (only available for the final burnup condition in [4]) is presented in the fifth column for comparison purposes. The symbol $\Delta$ is the difference between the value calculated in this study by SAMPLER and the reference value. Note that SD stands for 1 standard deviation and RSD is the relative standard deviation.

The difference between $k_{\text {inf }}$ mean value calculated by SAMPLER and the reference value was $0.4 \%$. It is typical difference that can be observed for criticality calculations of identical systems by different codes and XS libraries [11. This multiplication factor value is the average for the all the 1000 perturbed cases. As the XS perturbations are based on normal distribution,

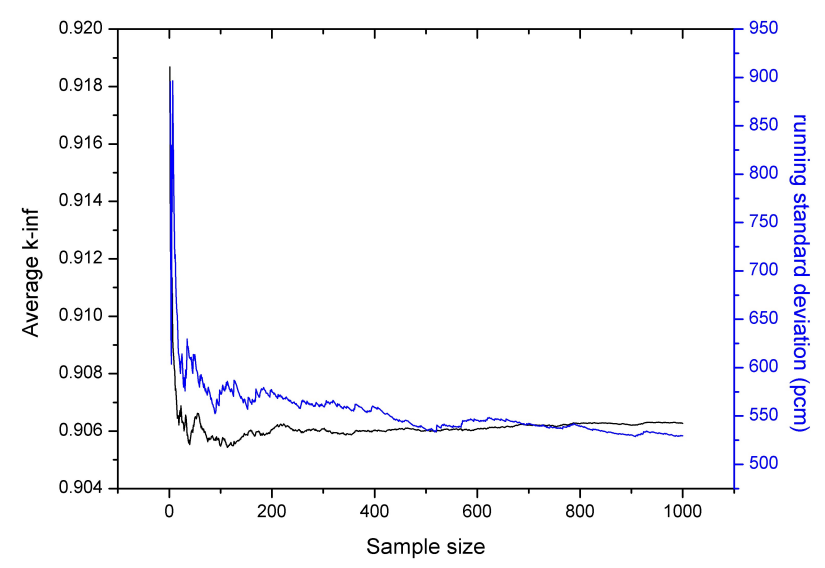

FiguRE 3 . Running $k_{\text {inf }}$ average and standard deviation as function of sample size for simulation on SAMPLER.

the mean value should stay almost the same as for a single case with average XS values. In order to provide information on sampling convergence, Fig. 3 presents the average $k_{\text {inf }}$ and the running standard deviation as function of the sample size. It is possible to see that after 800 samples, the average of calculated $k_{\text {inf }}$ mean values is stabilized.

In contrast to the above results, the increase of propagated uncertainty in $k_{\text {inf }}$ for burnup $60 \mathrm{GWd} / \mathrm{MTU}$ was $52 \%$. SAMPLER estimated 529 pcm for the standard deviation on $k_{\text {inf }}$ and the reference value is 805 pcm. The large difference (SCALE x UAM reference) can be explained by use of different versions of the covariance libraries.

There are also SAMPLER results for the fresh fuel and the final burnup. The estimated standard deviation on $k_{\text {inf }}$ was $770 \mathrm{pcm}$ for the fresh fuel and it decreased to $529 \mathrm{pcm}$ for the final burnup. The $31 \%$ difference comes from change of fuel composition during depletion. Each nuclide has its own XS covariance data thus the total uncertainty changes during depletion. The reason for the uncertainty decrease is discussed below and the main contributors to this phenomenon are illustrated in Fig. 4 and 5

It is possible to select the main contributors to the overall $k_{\text {inf }}$ uncertainty. These examples can also serve as the explanation for differences between the current and the older version of the XS covariance library in SCALE; and they are also the reason for computed uncertainty decrease during fuel depletion.

The usual main contributor to the calculation uncer- 


\begin{tabular}{lccccc}
\hline Variable & Distribution & Value & Std.dev & lower cutoff & upper cutoff \\
\hline Fuel_radius & normal & 0.46955 & $2.1667 \mathrm{e}-4$ & 0.4689 & 0.4702 \\
Gap_thickness & normal & 0.00955 & $1.3333 \mathrm{e}-3$ & 0.00555 & 0.01355 \\
Clad_thickness & normal & 0.0673 & $1.3333 \mathrm{e}-3$ & 0.0633 & 0.0713 \\
\hline
\end{tabular}

All dimensions in $\mathrm{cm}$.

TABLE 3. Parameters for variable sampling in SAMPLER input file.

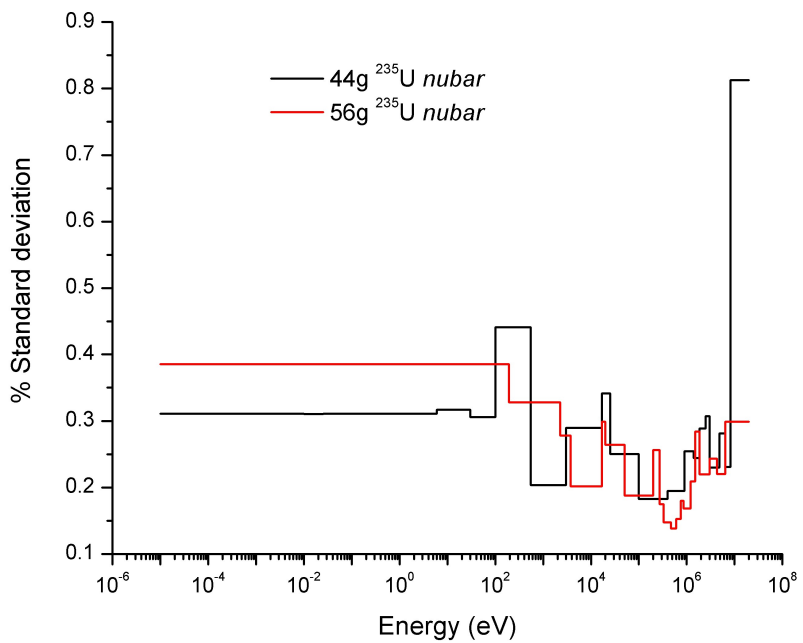

Figure $4 .{ }^{235} U$ nubar relative standard deviation by energy for covariance libraries 44groupcov and 56groupcov.

tainty is average fission neutron yield (nubar) of the major fissile nuclides, i.e. ${ }^{235} \mathrm{U}$ and ${ }^{239} \mathrm{Pu}$. Fig. 4 and Fig. 5 shows the relative standard deviation of this fission neutron yield in 44-groupcov and 56-groupcov for ${ }^{235} \mathrm{U}$ and ${ }^{239} \mathrm{Pu}$, respectively.

The relative standard deviation of ${ }^{235} U$ nubar below around $10 \mathrm{eV}$ is approximately by $24 \%$ higher for 56-groupcov. Much larger difference can be observed for ${ }^{239} P u$ nubar. Its RSD is about three times larger in 44-groupcov when compared to 56 -groupcov. It should be also noted that its uncertainty value is lower when compared to ${ }^{235} \mathrm{U}$ nubar. It is the reason for multiplication factor calculation uncertainty decrease during fuel depletion when adopting 56-groupcov.

In the final Figure 6, this comparison shows that the relative standard deviation of absorption in ${ }^{238} \mathrm{U}$ is comparable for these two covariance libraries and it is below $5 \%$ for all neutrons except fast neutrons.

In addition to the different covariance data used for the two methodologies compared, uncertainties may be introduced into the broad-group cross-sections due to approximations in the grouping procedure. In [12, it is showed that the dominant uncertainty is generally with regard to the energy weighting function used to average the point-wise data within a broader groups.

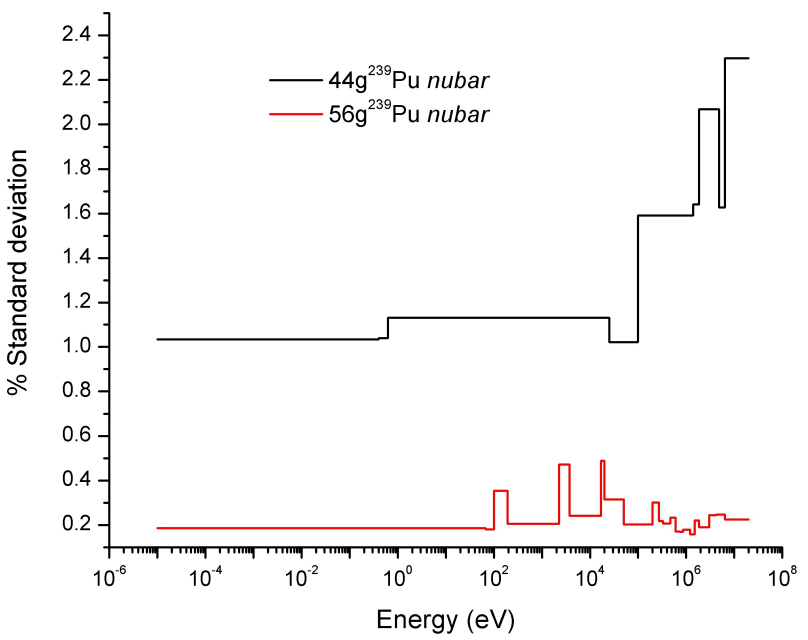

Figure 5. ${ }^{239} \mathrm{Pu}$ nubar relative standard deviation by energy for reproduced from covariance libraries 44 groupcov and 56groupcov.

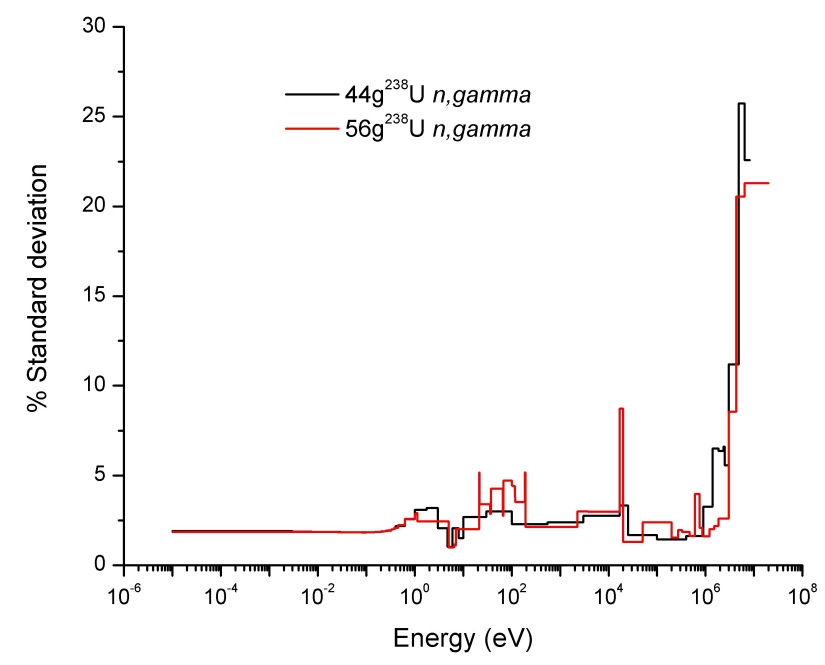

FiguRE $6 .{ }^{238} U(n, \gamma)$ relative standard deviation by energy for covariance libraries 44 groupcov and 56 groupcov.

\section{UNCERTAINTY ANALYSIS FROM GEOMETRY PERTURBATION}

The UAM benchmark model of PWR fuel rod presented in section 2.1 had its geometrical uncertain parameters of fuel radius, gap thickness, and clad thickness modeled in SAMPLER. The methodology adopted and resulting propagated uncertainty to the neutron multiplication factor are presented. 


\begin{tabular}{lcc|ccc|ccc}
\hline & & & \multicolumn{3}{c}{$0 \mathrm{GWd} / \mathrm{MTU}$} & \multicolumn{3}{c}{$60 \mathrm{GWd} / \mathrm{MTU}$} \\
Name & variable & \# samples & $k_{\text {inf }}$ & $R S D(\%)$ & $S D(\mathrm{pcm})$ & $k_{\text {inf }}$ & $R S D(\%)$ & $S D(\mathrm{pcm})$ \\
\hline Case1 & Fuel_radius & 300 & 1.40749 & 0.010 & 14 & 0.90494 & 0.002 & 2 \\
Case2 & Gap_thickness & 300 & 1.40749 & 0.004 & 6 & 0.90494 & 0.000 & 0 \\
Case3 & Clad_thickness & 300 & 1.40749 & 0.005 & 8 & 0.90494 & 0.001 & 1 \\
Case4 & All together & 300 & 1.40749 & 0.012 & 17 & 0.90494 & 0.003 & 3 \\
\hline
\end{tabular}

TABLE 4. Variables sampled for each study case and results of UQ with the contributors to uncertainty in $k_{\text {inf }}$.

\subsection{UNCERTAIN VARIABLES}

Normal distribution of the geometrical parameters was assumed. The UAM benchmark specifies values of standard deviations and there is also requirement to truncate the distribution at 3 standard deviations as lower cutoff and upper cutoff. Table 3 presents the parameters considered in the sampling of variables.

UQ for each variable was calculated in order to perform the sensitivity study of the geometric parameters. The values of fuel radius (fuelr), gap outer radius (gapr) and cladding outer radius (cladr) as function of the sampled variables (Tab. 3) are the following:

- Case1 (variable sampled: Fuel_radius )

fuelr $=$ Fuel_radius

gapr $=$ Fuel_radius +0.00955

cladr $=$ Fuel_radius $+0.00955+0.0673$

- Case2 (variable sampled: Gap_thickness)

fuelr $=0.46955$

gapr $=0.46955+$ Gap_thickness

cladr $=0.46955+$ Gap_thickness +0.0673

- Case3 (variable sampled: Clad_thickness)

fuelr $=0.46955$

gapr $=0.4791$

cladr $=0.4791+$ Clad_thickness

- Case4 (variable sampled: All together)

fuelr = Fuel radius

gapr $=$ Fuel_radius + Gap_thickness

cladr = Fuel_radius + Gap_thickness

+ Clad_thickness

Since the cladding outer radius (cladr) depends on uncertain variables values, the moderator/fuel volume ratio and the cladding density vary according to the sampled values. However, for being a neutron nonabsorbing material (Zircaloy-4), the density change was disregarded.

The fuel meat density (D), dependent on fuel radius for Case 1 and Case 4, is updated according to equation 1. This allows to keep the value of fuel mass constant when fuel radius sampled value is updated in the model geometry. The nominal density is worth $10.283 \mathrm{~g} / \mathrm{cm}^{3}$ according to Tab. 1

$$
D_{\text {new }}\left[\mathrm{g} / \mathrm{cm}^{3}\right]=10.283 \times\left(\frac{0.46955}{\text { Fuel_radius }}\right)^{2}
$$

\subsection{Results}

The cases considered are presented in Tab. 4, where the variable under study is given in the second column. The contribution to uncertainty in $k_{\text {inf }}$ are presented for $0 \mathrm{GWd} / \mathrm{MTU}$ and $60 \mathrm{GWd} / \mathrm{MTU}$ burnups. Uncertainty in the fuel radius (Case 1) is the biggest contributor to the overall calculation uncertainty due to the fact that its value directly influences fuel volume. The second in rank is the clad thickness represented by Case 3. It was noticed that the effect of uncertainties in $k_{\text {inf }}$ is much more pronounced for the fresh fuel rather than for the final burnup condition.

\section{Conclusions}

Uncertainty propagation analysis was performed for the burnup pin-cell exercise I-b of the UAM benchmarks. The SAMPLER code using SCALE 6.2 56groupcov covariance library had the results compared against participant-averaged result from the benchmark exercise I-b. The difference between propagated uncertainty (SD) in $k_{\text {inf }}$ calculated by SAMPLER and the reference case (UAM benchmark) was $52 \%$. Such difference is expected since the codes used different versions of covariance libraries with different group structure.

Comparison of results for the fresh and spent fuel for the same covariance library was done for the SAMPLER results alone, as there are no benchmark reference results. The observed $31 \%$ difference in standard deviations is attributed to the fact that propagated uncertainty is mainly affected by nuclide composition that changes along the burnup. Future research must include other nuclear libraries for comparison.

When propagating geometrical uncertainties, the effect of uncertainty in fuel radius, clad and gap thickness was calculated. It was noted that the influence of geometric input uncertainties on $k_{\text {inf }}$ is more pronounced for the fresh than for the depleted fuel. This can be explained by the fact that fresh fuel contains higher density of fissile material and influences more the neutron population of the reactor. The sensitivity study also confirmed that uncertainty of fuel radius (Case 1) is the biggest contributor to the overall calculation uncertainty since it influences directly fuel volume even though the total fuel mass is preserved. This study also confirmed that the contribution of manufacturing geometrical uncertainties to the prediction 
of $k_{\text {inf }}$ is smaller than the contribution of cross-section uncertainties.

\section{ACKNOWLEDGEMENTS}

The mobility of the Brazilian co-author to Department of Nuclear Reactors, Czech Technical University in Prague has been possible with the Erasmus Mundus SMART ${ }^{2}$ support (Project Reference: 552042-EM-1-2014-1-FR-ERA MUNDUS-EMA2) coordinated by Centrale Supelec.

\section{REFERENCES}

[1] E. B.T. Rearden, M.A. Jessee. SCALE Code System Version 6.2.1. Tech. Rep. ORNL/TM-2005/39, Oak Ridge National Laboratory, Oak Ridge, Tennessee, 2016.

[2] A. Haldar, S. Mahadevan. Probability, reliability, and statistical methods in engineering design. John Wiley, 2000 .

[3] K. Ivanov, M. Avramova, S. Kamerow, et al. Benchmark for uncertainty analysis in modeling (UAM) for design, operation and safety analysis of LWRs, vol. 1. Citeseer, 2013.

[4] R. N. Bratton, M. Avramova, K. Ivanov. OECD/NEA benchmark for uncertainty analysis in modeling (UAM) for LWRs-summary and discussion of neutronics cases (phase I). Nuclear Engineering and Technology 46(3):313-342, 2014. DOI:https://doi.org/10.5516/NET.01.2014.710

[5] M. Chadwick, M. Herman, P. Obložinskỳ, et al. ENDF/B-VII. 1 nuclear data for science and technology: cross sections, covariances, fission product yields and decay data. Nuclear Data Sheets 112(12):2887-2996, 2011. DOI:https://doi.org/10.1016/j.nds.2011.11.002
[6] L. Fiorito, D. Piedra, O. Cabellos, C. Diez. Inventory calculation and nuclear data uncertainty propagation on light water reactor fuel using ALEPH-2 and SCALE 6.2. Annals of Nuclear Energy 83:137-146, 2015. DOI:https://doi.org/10.1016/j.anucene.2015.03.046

[7] W. Wieselquist, T. Zhu, A. Vasiliev, H. Ferroukhi. PSI methodologies for nuclear data uncertainty propagation with CASMO-5M and MCNPX: Results for OECD/NEA UAM benchmark Phase I. Science and Technology of Nuclear Installations 2013, 2013. DOI:http://dx.doi.org/10.1155/2013/549793

[8] A. Yankov, B. Collins, M. Klein, et al. A two-step approach to uncertainty quantification of core simulators. Science and Technology of Nuclear Installations 2012, 2012. DOI:http://dx.doi.org/10.1155/2012/767096

[9] O. Cabellos. Presentation and Discussion of the UAM/Exercise I-1b:"Pin-Cell Burn-Up Benchmark" with the Hybrid Method. Science and Technology of Nuclear Installations 2013, 2013. DOI:http://dx.doi.org/10.1155/2013/790206

[10] A. Aures, F. Bostelmann, M. Hursin, O. Leray. Benchmarking and application of the state-of-the-art uncertainty analysis methods XSUSA and SHARK-X. Annals of Nuclear Energy 101:262-269, 2017. DOI:https://doi.org/10.1016/j.anucene.2016.11.025

[11] E. Fridman, J. Leppänen. On the use of the serpent monte carlo code for few-group cross section generation. Annals of Nuclear Energy 38(6):1399 - 1405, 2011. DOI:https://doi.org/10.1016/j.anucene.2011.01.032

[12] Z. Ting. Sampling-Based Nuclear Data Uncertainty Quantification for Continuous Energy Monte Carlo Codes. Ph.D. thesis, École Polytechnique Féeérale de Lausanne, 2015. DOI:10.5075/epfl-thesis-6598 